\title{
Electromyography Low Pass Filtering Effects on the Classification of Hand Movements in Amputated Subjects
}

\author{
Manfredo Atzori \\ Information Systems Institute, University of Applied Sciences Western Switzerland (HES-SO Valais), Sierre, \\ Switzerland \\ Email: manfredo.atzori@hevs.ch \\ Henning Müller \\ Information Systems Institute, University of Applied Sciences Western Switzerland (HES-SO Valais), Sierre, \\ Switzerland \\ Email: henning.mueller@hevs.ch
}

\begin{abstract}
People with transradial hand amputations can have control capabilities of prosthetic hands via surface electromyography (sEMG) but the control systems are limited and usually not natural. In the scientific literature, the application of pattern recognition techniques to classify hand movements in SEMG led to remarkable results but the evaluations are usually far from real life applications with all uncertainties and noise. Therefore, there is a need to improve the movement classification accuracy in real settings.

Smoothing the signal with a low pass filter is a common pre- processing procedure to remove high-frequency noise. However, the filtering frequency modifies the signal strongly and can therefore affect the classification results. In this paper we analyze the dependence of the classification accuracy on the pre-processing low-pass filtering frequency in 3 hand amputated subjects performing $\mathbf{5 0}$ different movements. The results highlight two main interesting aspects. First, the filtering frequency strongly affects the classification accuracy, and choosing the right frequency between $1 \mathrm{~Hz}-5 \mathrm{~Hz}$ can improve the accuracy up to $5 \%$. Second, different subjects obtain the best classification performance at different frequencies. Theoretically these facts could affect all the similar classification procedures re- ducing the classification uncertainity. Therefore, they contribute to set the field closer to real life applications, which could deeply change the life of hand amputated subjects.
\end{abstract}

Index Terms - surface electromyography, signal filtering, machine learning, rehabilitation engineering

\section{INTRODUCTION}

Hand prostheses controlled by surface electromyography (sEMG) have been used since the late 1960s [1]. However, they still have several important limits. First, usually they offer only 2 or 3 degrees of freedom and the number of movements that the subjects can perform is therefore limited (usually opening and closing of the prosthesis). The number of movements can be increased using specific control sequences but in these cases the movements are far from being natural and easy to be reproduced. Second, the control systems are not "natural", which means that the movement that the amputee is doing with the intact hand is different from the movement performed by the prosthesis. Third, the prostheses require long and complicated training procedures. These facts contribute to the limited use of sEMG prostheses [2].

In the scientific literature, several control schemes based on classifiers have been proposed to solve these control problems [3], [4], [5], [6]. However, these results are still far from the possibility of being applied in practice as any misclassification can have a negative effect. Therefore, there is a clear need to improve the movement classification accuracy.

Smoothing the rectified signal with a low pass filter is a common pre-processing procedure to remove highfrequency noise components [7]. However, the filtering frequency strongly modifies the signal and can affect the classification results.

In this paper we analyze the dependence of the classification accuracy on the pre-processing low-pass filtering frequency in 3 hand amputated subjects performing 50 different movements. Also, for each subject and for each frequency we find a selection of up to 15 independent movements that can be perfectly discriminated. The datasets come from the NinaPro (Non-Invasive Adaptive Hand Prosthetics) project [8], which has the aim to help the scientific progress in the field of sEMG movement recognition with a benchmark database to develop, test and compare machine learning algorithms. Currently, two databases with 27 and 40 intact subjects using different electrodes and with slightly over 50 movements can be downloaded from the project website (http://ninaweb.hevs.ch/). The used sEMG setup is standard and the classification procedure is fast. The results highlight that choosing the right filtering 
frequency can improve the accuracy and that different subjects obtain the best classification performance at different frequencies. These facts should affect all similar classification procedures, reducing the classification uncertainity. Therefore they contribute to set the field closer to real life applications, which could deeply change the life of hand amputated subjects.

\section{METHODS}

\section{A. Data Acquisition}

The datasets used in this paper were acquired from three subjects with a transradial amputation of the right forearm. The amputations are transradial medium and long below the elbow, with a remaining percentage of the forearm between $70 \%$ and $90 \%$. The subjects are male, right handed and their clinical characteristics are described in Table I.

TABLE I ClinicAl Data OF HAND AMPUTATED SUBJECTS

\begin{tabular}{|c|l|l|l|l|l|}
\hline Subject & Age & $\begin{array}{l}\text { Missing } \\
\text { Hand }\end{array}$ & $\begin{array}{l}\text { Years from } \\
\text { Amputation }\end{array}$ & $\begin{array}{l}\text { Remaining } \\
\text { Forearm } \\
\text { Percentage }\end{array}$ & $\begin{array}{l}\text { Number } \\
\text { Movement } \\
\text { s }\end{array}$ \\
\hline 1 & 35 & Left & 6 & 70 & 50 \\
\hline 2 & 44 & Right & 14 & 90 & 50 \\
\hline 3 & 55 & Right & 5 & 90 & 50 \\
\hline
\end{tabular}

The sEMG data were acquired according to the final version of the NinaPro acquisition protocol [8], [9], [10]. The protocol includes 6 repetitions of 50 movements (Figure 1), selected from the hand taxonomy and robotics literature, (e.g., [11], [12], [13], [14]). During the acquisition, the amputated subjects were asked to think to repeat the movements shown on the screen of a laptop according to a bilateral imitation procedure [3]. Each movement repetition lasted 5 seconds and was followed by 3 seconds of rest.

The muscular activity was recorded at $2 \mathrm{kHz}$ using 12 active double-differential wireless electrodes from a DelsysTrigno Wireless EMG system. The electrodes were positioned as shown in Figure 2: eight electrodes were equally spaced around the forearm in correspondence to the radio humeral joint; two electrodes were placed on the main activity spots of the flexor digitorum and of the extensor digitorum as described in [8]; two electrodes were placed on the main activity spots of the biceps and of the triceps. The described locations have been chosen in order to combine a dense sampling approach [15], [16], [17] with a precise anatomical positioning strategy [18], [19]. Moreover, such a setup permits the use of spatial registration algorithms [20] to improve the classification results. The electrodes were fixed on the forearm using their standard adhesive bands. A hypoallergenic elastic latex-free band was placed around the electrodes to keep them fixed during the acquisition.

\section{B. Data Analysis}

1) Preprocessing: First, all the data were synchronized by linearly interpolating them to the highest recording frequency (i.e., $2 \mathrm{kHz}$ ). Second, the sEMG was low-pass filtered using a zero-phase second order Butterworth filter at different frequencies in order to remove highfrequency noise components and to analyze the effect of each frequency on the movement classification. The used frequencies are the following: $0.1,0.25,0.5,0.75,1,2,3$, $4,5,10,15,25,50,100,200 \mathrm{~Hz}$. The second order Butterworth filter was used in accordance to common preprocessing in hand movements sEMG literature [3], [8]. Then, the signal from each repetition of each movement was segmented with a Generalized Likelihood Ratio approach [21], which realigns the movement labels to time windows that contain increased sEMG activity. Finally, the data of all the movement repetitions were normalized to the same time length and the signal was normalized to its maximum and divided by the standard deviation.

2) Classification: The classification procedure is balanced and it is an evolution of the one described in [22]. For each filtering frequency, a Distance-based Decision Classifier (DDC) [23] based on the normalized Euclidean distance was applied to each repetition of all the movements with a leave one out approach (i.e. one sample for testing, five samples for training). The DDC was chosen because it is very fast (the classification of each movement repetition requires approximately $60 \mathrm{~ms}$ using Matlab with a non optimized procedure on a $2.7 \mathrm{GHz}$ Macbook pro) and it gives good results in this kind of tasks (it outperforms $\mathrm{k}-\mathrm{NN}$ algorithms in most experiments and the results are usually comparable to or better than SVMs [23]). Finally, for each filtering frequency, the same classification procedure was applied recursively to subsets of movements in order to find for each subject a subset of independent movements that does not present any misclassification. In this way the complexity of the task is reduced but it is possible to show that for fewer movements a very high classification accuracy is possible without training the subject.

\section{RESULTS}

The classification accuracy and the number of independent movements identified for the three amputated subjects for each considered frequency are shown respectively in Figure 3 and in Figure 4. It can be noticed how in all three subjects the classification accuracy increases up to its maximum between 0 and $3 \mathrm{~Hz}$, and then it starts to slowly decrease. We obtained a similar (but less evident) result also for the set of independent movements.

The best classification results for the subjects are summarized in Table II and in Figure 5. The results were obtained with a $3 \mathrm{~Hz}$ filtering frequency on subject 1 , and with a $1 \mathrm{~Hz}$ filtering frequency on subjects 2 and 3. Two subjects obtained the highest classification accuracy, $61.78 \%$. The Gaussian fit of the movements' maximal classification results leads to a mean of $51.62 \%$, which is more than 25 times the chance level for 50 movements $(2 \%)$, and is well fitted by a Gaussian distribution $(p<0.05)$ Figure 5 . The average number of independent movements is 9.33 , with a maximum of 12 


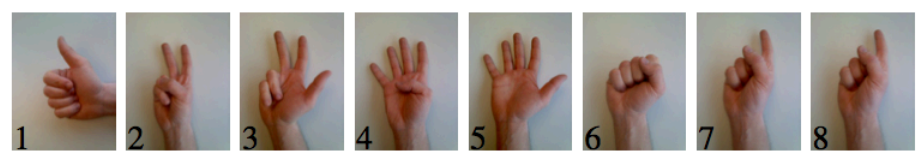

(a) Isometric, isotonic hand configurations.
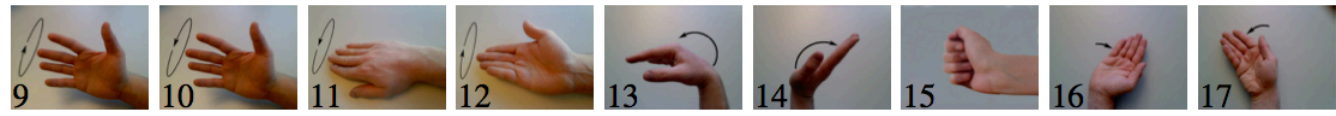

(b) Basic movements of the wrist.
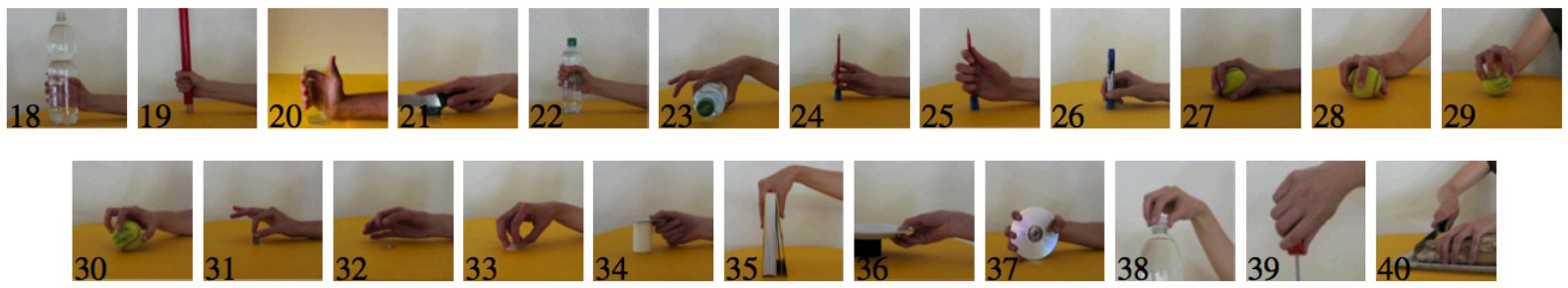

(d) Grasps and functional movements.
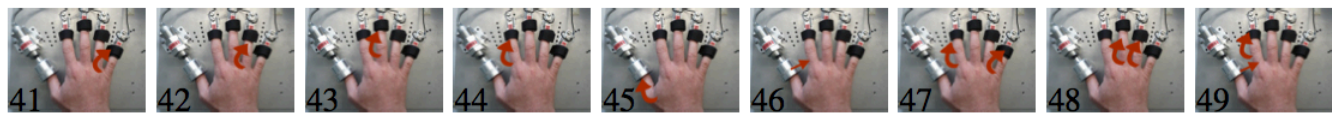

(e) Single and Multiple Fingers Force Measurement Patterns

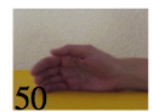

(f) Rest posi-

tion.

Figure 1 The 50 movements acquired within the NinaPro acquisition protocol.

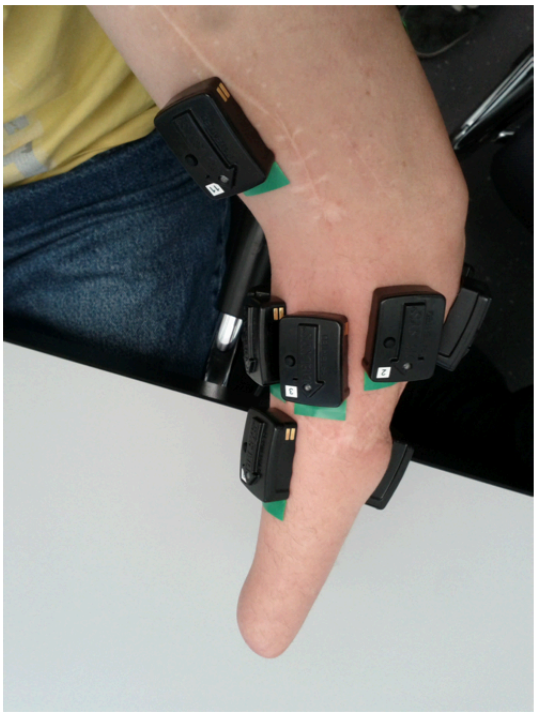

(a)

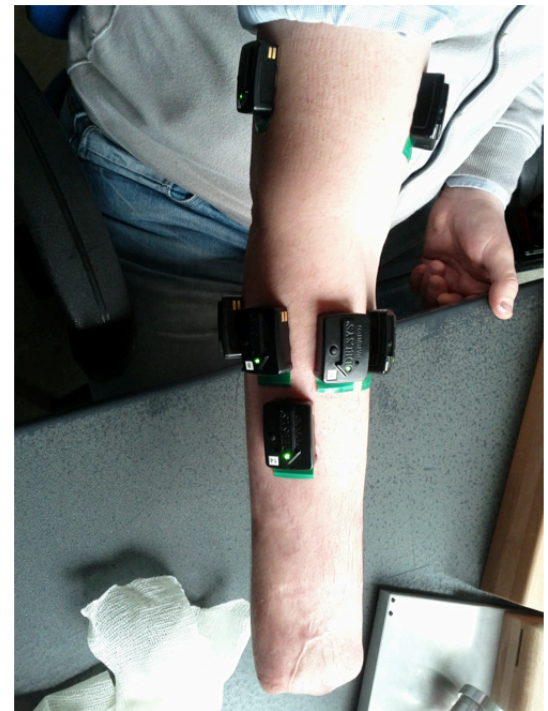

(b)

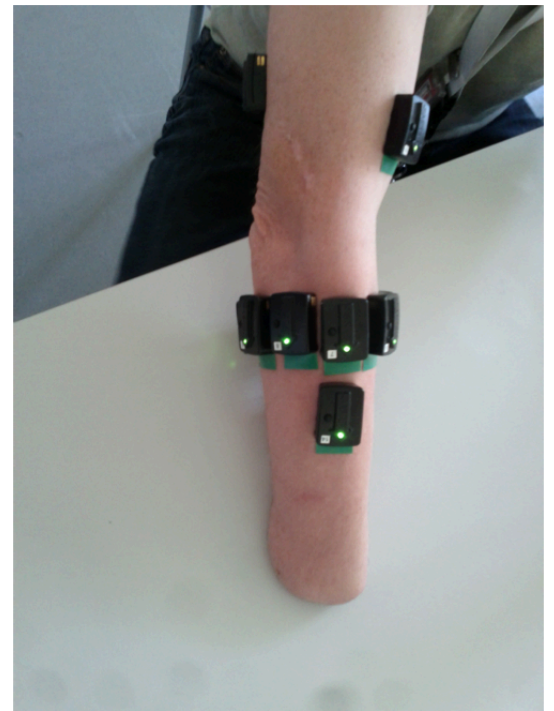

(c)

Figure 2 Forearm of the transradial amputated subjects: (a) subject 1; (b) subject 2; (c) subject 3. 
movements for subject 1. Different subsets of movements could also be selected on the basis of other parameters, such as the functional usefulness of the movements.

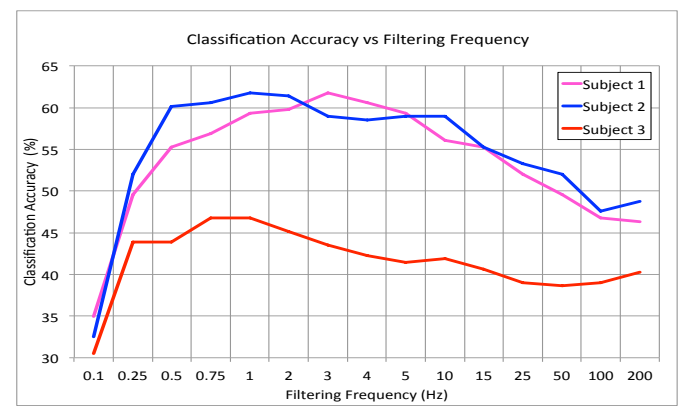

Figure 3 Filtering frequency effect on the classification accuracy.

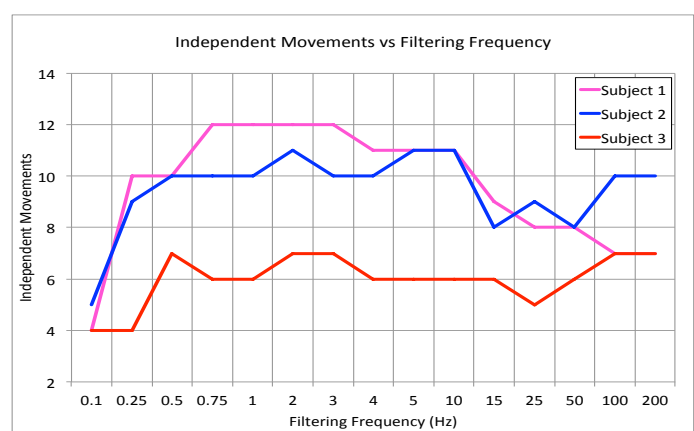

Figure 4 Filtering frequency effect on the number of independent movements.

TABLE II ClASSIFICATION RESUlTS FOR HAND AMPUTATED SUBJECTS

\begin{tabular}{|c|c|c|c|}
\hline Subject & $\begin{array}{c}\text { Frequency } \\
\text { (Hz) }\end{array}$ & $\begin{array}{c}\text { Classification } \\
\text { Accuracy }\end{array}$ & $\begin{array}{c}\text { Independent } \\
\text { Movements }\end{array}$ \\
\hline 1 & 3 & $61.78 \%$ & 12 \\
\hline 2 & 1 & $61.78 \%$ & 10 \\
\hline 3 & 1 & $46.74 \%$ & 6 \\
\hline
\end{tabular}

In order to get a deeper perspective of the independent movement selection, in Figure 6 we present a statisticalevaluation of the identified independent movements in the three subjects. It can be noticed that only 5 movements are repeated in more than two different subjects, which means that the movements are usually different in different subjects.

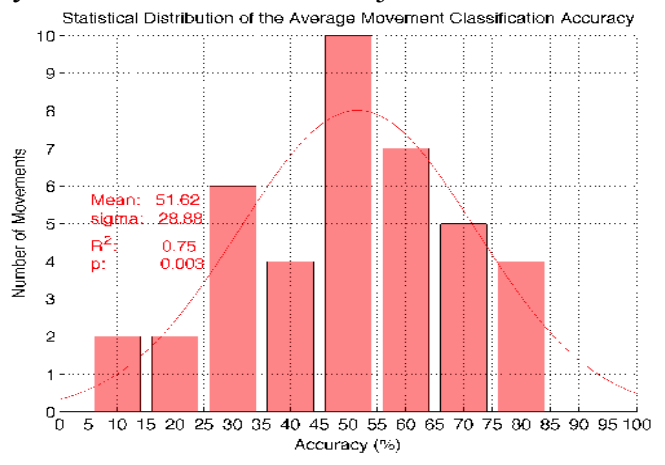

Figure 5 Distribution and Gaussian fit of all the movement classification results in hand amputated subjects.

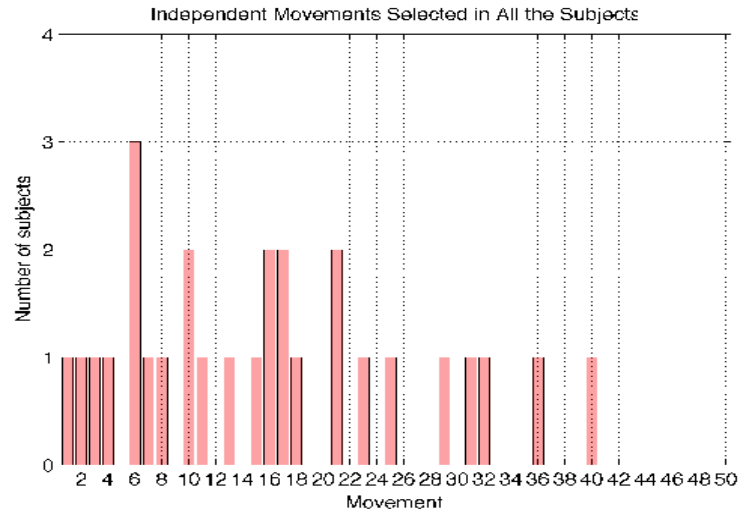

Figure 6 Generalization of independent movements.

\section{CONCLUSION}

Currently, myoelectric prostheses permit hand amputated subjects to perform few simple movements.

However, the control possibilities are still limited and not natural. In the scientific literature, the application of pattern recognition techniques to classify hand movements in sEMG led to remarkable results. However, the results are still not accurate enough to permit real life applications as small mistakes can have important consequencies. Therefore, there is a need to improve the movement classification accuracy. Smoothing the signal with a low pass filter is a common pre-processing procedure in sEMG to remove high-frequency noise components. However, the filtering frequency modifies the signal strongly and can therefore affect the classification results.

In this paper we analyze the dependence of the classification accuracy on the pre-processing low-pass filtering frequency in 3 hand amputated subjects performing 50 movements. The subjects have respectively $70 \%, 90 \%$ and $90 \%$ of the forearm remaining. The datasets are from the NinaPro database, which was developed in order to overcome the limits of dexterous prosthetics through the evaluation of machine learning algorithms from the worldwide scientific community on a common database.

The results highlight four main interesting aspects. First, the filtering frequency strongly affects the classification accuracy (Figure 3). In all subjects the classification accuracy increases up to its maximum between 0 and $3 \mathrm{~Hz}$, and then it starts to slowly decrease. Choosing the right frequency between $1 \mathrm{~Hz}-5 \mathrm{~Hz}$ can improve the accuracy by up to $5 \%$. A similar (but less evident) result was obtained also for the set of independent movements. Second, although the trends of the classification performance are similar in all the subjects, different subjects obtain the best classification performances at different frequencies. The described results could theoretically affect most of the sEMG classification procedures that use low-pass filtering before classification. Therefore, the optimization for each subject of the pre-processing filtering frequency could lead to an overall improvement of the sEMG movement classification performance. Third, the ratio between the accuracy and the chance level (more than 25 times) is 
very high in comparison to other results described in the literature for similar tasks, e.g. 5.7 [3] (6 movements, accuracy 95\%), 8.5 [24] (10 movements, accuracy 84.4\%), 10.56 [16] (12 movements, accuracy 87.8\%). Fourth, the results on the selection of in- dependent movements for the transradial amputated subjects (Table II, Figure 6) highlights the possibility for the amputated subjects to control a robotic prosthetic hand with up to 12 different movements with $60 \mathrm{~ms}$ of computational response time (i.e., in a time that would be realistic for use in everyday life). It has to be noticed that different subsets of movements could also be selected on the basis of other parameters, such as the functional usefulness of the movements. In conclusion, the results are an important step towards the natural control of dexterous prosthetic hands and they contribute to set the field closer to real life applications, which could deeply change the life of hand amputated subjects.

\section{ACKNOWLEDGMENT}

The authors would like to thank the intact and hand amputated subjects that volunteered for this project and the Swiss National Science Foundation that supports this work through the Sinergia project NINAPRO (NonInvasive Adaptive Prosthetics).

\section{REFERENCES}

[1] F. R. Finley and R. W. Wirta, "Myocoder studies of multiple myopotential response," Archives of Physical Medicine and Rehabilitation, vol. 48, no. 11, pp. 598-601, 1967.

[2] D. J. Atkins, "Epidemiologic overview of individuals with upper-limb loss and their reported research priorities," Journal of Prosthetics \& Orthotics, vol. 8, no. 1, pp. 2-11, 1996.

[3] C. Castellini, E. Gruppioni, A. Davalli, and G. Sandini, "Fine detection of grasp force and posture by amputees via surface electromyography," Journal of Physiology (Paris), vol. 103, no. 3-5, pp. 255-262, 2009.

[4] T. R. Farrell and R. F. Weir, "A comparison of the effects of electrode implantation and targeting on pattern classification accuracy for pros- thesis control," IEEE Transactions on Biomedical Engineering, vol. 55, pp. 2198-2211, March 2008.

[5] B. Crawford, K. Miller, P. Shenoy, and R. Rao, "Real-time classification of electromyographic signals for robotic control," in Proc. of AAAI, 2005, pp. 523-528.

[6] B. Peerdeman, D. Boere, H. Witteveen, R. H. in 't Veld, H. Hermens, S. Stramigioli, H. Rietman, P. Veltink, and S. Misra, "Myoelectric forearm prostheses: State of the art from a usercentered perspective," Journal of Rehabilitation Research and Development, vol. 48, no. 6, pp. 719-738, 2011.

[7] R. Merletti and P. Di Torino, "Standards for reporting emg data," J ElectromyogrKinesiol, vol. 9, no. 1, pp. 3-4, 1999.

[8] M. Atzori, A. Gijsberts, I. Kuzborskij, S. Heynen, A.-G. M. Hager, O. Deriaz, C. Castellini, H. Müller, and B. Caputo, "Characterization of a benchmark database for myoelectric movement classification," IEEE Transactions on Neural Systems and Rehabilitation Engineering, 2014, (in press).

[9] A. Gijsberts, M. Atzori, C. Castellini, H. Müller and B. Caputo, "The movement error rate for evaluation of machine learning methods for semg-based hand movement classification," IEEE Transactions on Neural Systems and Rehabilitation Engineering, 2013.

[10] A. Gijsberts and B. Caputo, "Exploiting accelerometers to improve movement classification for prosthetics," in Proc. of IEEE International Conference on Rehabilitation Robotics (ICORR), 2013.

[11] T. Feix, "Grasp taxonomy comparison," Otto Bock GmbH, Tech. Rep., 2008. [Online]. Available: http://grasp.xief.net
[12] M. R. Cutkosky, "On grasp choice, grasp models, and the design of hands for manufacturing tasks," IEEE Transactions on Robotics and Automation, vol. 5, no. 3, pp. 269-279, June 1989.

[13] N. Kamakura, M. Matsuo, H. Ishii, F. Mitsuboshi and Y. Miura, "Patterns of static prehension in normal hands," The American journal of occupational therapy: official publication of the American Occupational Therapy Association, vol. 34, no. 7, pp. 437-445, 1980.

[14] S. J. Edwards, D. J. Buckland and J. D. McCoy-Powlen, Developmental and Functional Hand Grasps. Slack Incorporated, 2002.

[15] O. Fukuda, T. Tsuji, M. Kaneko, and A. Otsuka, "A humanassisting manipulator teleoperated by EMG signals and arm motions," IEEE Transactions on Robotics and Automation, vol. 19, no. 2, pp. 210-222, April 2003.

[16] F. V. Tenore, A. Ramos, A. Fahmy, S. Acharya, R. EtienneCummings, and N. V. Thakor, "Decoding of individuated finger movements using surface electromyography," IEEE Transactions on Biomedical Engineering, vol. 56, no. 5, pp. 1427-1434, 2009.

[17] C. Castellini and P. van der Smagt, "Surface EMG in advanced hand prosthetics," Biological Cybernetics, vol. 100, no. 1, pp. $35-47,2009$.

[18] C. J. D. Luca, "The use of surface electromyography in biomechanics," Journal of Applied Biomechanics, vol. 13, no. 2, pp. 135-163, 1997.

[19] C. Castellini, A. E. Fiorilla and G. Sandini, "Multi-subject / daily- life activity EMG-based control of mechanical hands," Journal of Neuroengineering and Rehabilitation, vol. 6, no. 41, 2009.

[20] M. Atzori, C. Castellini and H. Müller, "Spatial registration of hand muscle electromyography signals," in 7th International Workshop on Biosignal Interpretation, Como, July 2012.

[21] I. Kuzborskij, A. Gijsberts and B. Caputo, "On the challenge of classifying 52 hand movements from surface electromyography," in Proc. of EMBC - the 34th annual conference of the IEEE Engineering in Medicine and Biology Society, 2012, pp. 49314937.

[22] Atzori, M. Baechler and H. Müller, "Recognition of hand movements in a trans-radial amputated subject by sEMG," in Proc. of IEEE International Conference on Rehabilitation Robotics (ICORR), 2013.

[23] J. Hamidzadeh, R. Monsefi, and H. S. Yazdi, "DDC: distancebased decision classifier," Neural Computing and Applications, vol. 21, no. 7, pp. 1697-1707, 2012.

[24] G. Li, A. E. Schultz and T. A. Kuiken, "Quantifying pattern recognition-based myoelectric control of multifunctional transradial prostheses," IEEE Trans Neural SystRehabilEng, vol. 18, no. 2, pp. 185-92, Apr 2010.

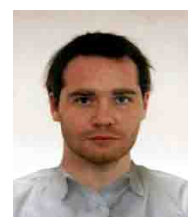

Manfredo Atzorireceived a M.Sc. degree in Physics at the University of Padova, Padova, Italy, in 2006, and a Ph.D. degree in Bioengineering at the University of Padova in 2009. From 2006 to 2010 he was a researcher at the Brain Imaging research unit of the University of Verona, Verona, Italy, and he collaborated on several research projects on the quantitative analysis of medical images with functional and structural techniques. Since 2011 he is postdoc at the Medgift Unit of the University of Applied Sciences Western Switzerland (HES-SO Valais), where he works within the Ninapro Project on the acquisition and the analysis of surface electromyography data to control upper-limb dexterous robotic prostheses using modern machine learning techniques.

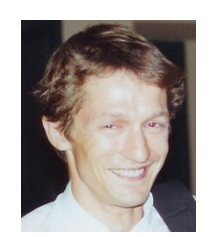

Henning Müller Henning Müller studied medical informatics at the University of Heidelberg, Germany, then worked at Daimler-Benz research in Portland, OR, USA. From 1998-2002 he worked on his $\mathrm{PhD}$ degree at the University of Geneva, Switzerland with a research stay at Monash University, Melbourne, Australia. Since 2002 Henning has been working for medical informatics at the University hospital of Geneva, where he was named titular professor in medicine 
in 2014. Since 2007 he has also been a full professor at the HES-SO Valais and since 2011 he is responsible for the eHealth unit of the school. Henning is coordinator of the Khresmoi EU project, scientific coordinator of the VISCERAL EU project and initiator of the
ImageCLEF benchmark. He has worked on several other EU projects that include the access to and the analysis of medical data. He has authored over 400 scientific papers and is in the editorial board of several journals. 